\title{
JH gene sequence analysis in a patient with Waldenström's macroglobulinaemia with subsequent development of immunoblastic lymphoma
}

\author{
H Kondo, N Yumoto, A Mikata, Y Date
}

\begin{abstract}
A case of Waldenström's macroglobulinaemia with subsequent development of immunoblastic lymphoma in a 69 year old man is reported. Plasmacytoid lymphocytes were initially observed in both peripheral blood and bone marrow smears. Lymph node biopsy was interpreted as malignant lymphoma, diffuse mixed cell type compatible with Waldenström's macroglobulinaemia. Complete remission resulted on combined treatment with prednisolone and melphalan. Four years later, lymphadenopathy recurred and biopsy revealed the development of an immunoblastic lymphoma. Initially, this lymphoma was thought to be the malignant transformation of Waldenström's microglobulinaemia, because both tumours produced IgM ( $\kappa$ light chains). Sequencing of immunoglobulin heavy chain genes of Waldenström's macroglobulinaemia and immunoblastic lymphoma cells, however, revealed different DH and JH usage between the two, indicating the independent nature of the two haematological malignancies. These results indicate that clonality of primary and secondary tumours should be determined not by phenotypic, but by genotypic analysis.
\end{abstract}

(f Clin Pathol: Mol Pathol 1995;48:M215-M218)

Keywords: Waldenström's macroglobulinaemia, immunoblastic lymphoma, transformation, JH gene sequence analysis.

First Department of Pathology, Chiba University School of Medicine, Chiba, Japan

N Yumoto

A Mikata

Correspondence to:

Dr $\mathrm{H}$ Kondo, Division of Haematology and Oncology,

Department of Medicine, Shimizu Kousei Hospital, 578-1 Ihara-cho,

Shimizu City,

Shizuoka 424-01,

Japan.

Accepted for publication 24 April 1995 immunoblastic lymphoma have similar B cel characteristics, suggesting that these tumours have a common clonal origin. However, the assumption that the presence of the same immunoglobulin heavy and light chains is synonymous with clonality is an over simplification, as many different B cell malignancies express the same immunoglobulin heavy and light chains. Furthermore, it is postulated that additional phenotypic characterisation with monoclonal antibodies specific for B cell markers provides conclusive proof of the clonal identify of the malignancies; however, this merely illustrates the stage of the tumour cells and not their clonal identity. Therefore, the clonal differences/similarities between two $B$ cell malignancies should be evaluated by immunoglobulin gene rearrangement analysis.

Here, we report a case of Waldenström's macroglobulinaemia where the patient developed immunoblastic lymphoma in the course of four years of treatment with prednisolone and melphalan.

\section{Case report}

A 64 year old man was admitted to hospital in May 1989 with hepatosplenomegaly and systemic lymphadenopathy. Haematological analysis on admission revealed anaemia and leucocytosis with a leucocyte count of $11 \cdot 1 \times 10^{9} / 1$. Peripheral blood smears showed $60 \%$ lymphoplasmacytoid cells. Serum protein was $12.6 \mathrm{~g} / \mathrm{dl}$ and immunoelectrophoresis demonstrated that the monoclonal protein was IgM ( $\kappa$ light chains) with a serum concentration of $19590 \mathrm{mg} / \mathrm{dl}$. The bone marrow was infiltrated by lymphoplasmacytoid cells. Microscopic examination of a lymph node revealed malignant lymphoma, mixed large and small plasmacytoid cells of B cell phenotype and mature plasma cells. A diagnosis of Waldenström's macroglobulinaemia was reached. The patient was treated with melphalan and prednisolone for three and a half years. His general condition improved and the concentration of serum $\operatorname{IgM}$ decreased to within the normal range following treatment.

The patient was readmitted because of recurrent lymphadenopathy four years after the initial diagnosis. There were no abnormal haematological data except for slight anaemia. 

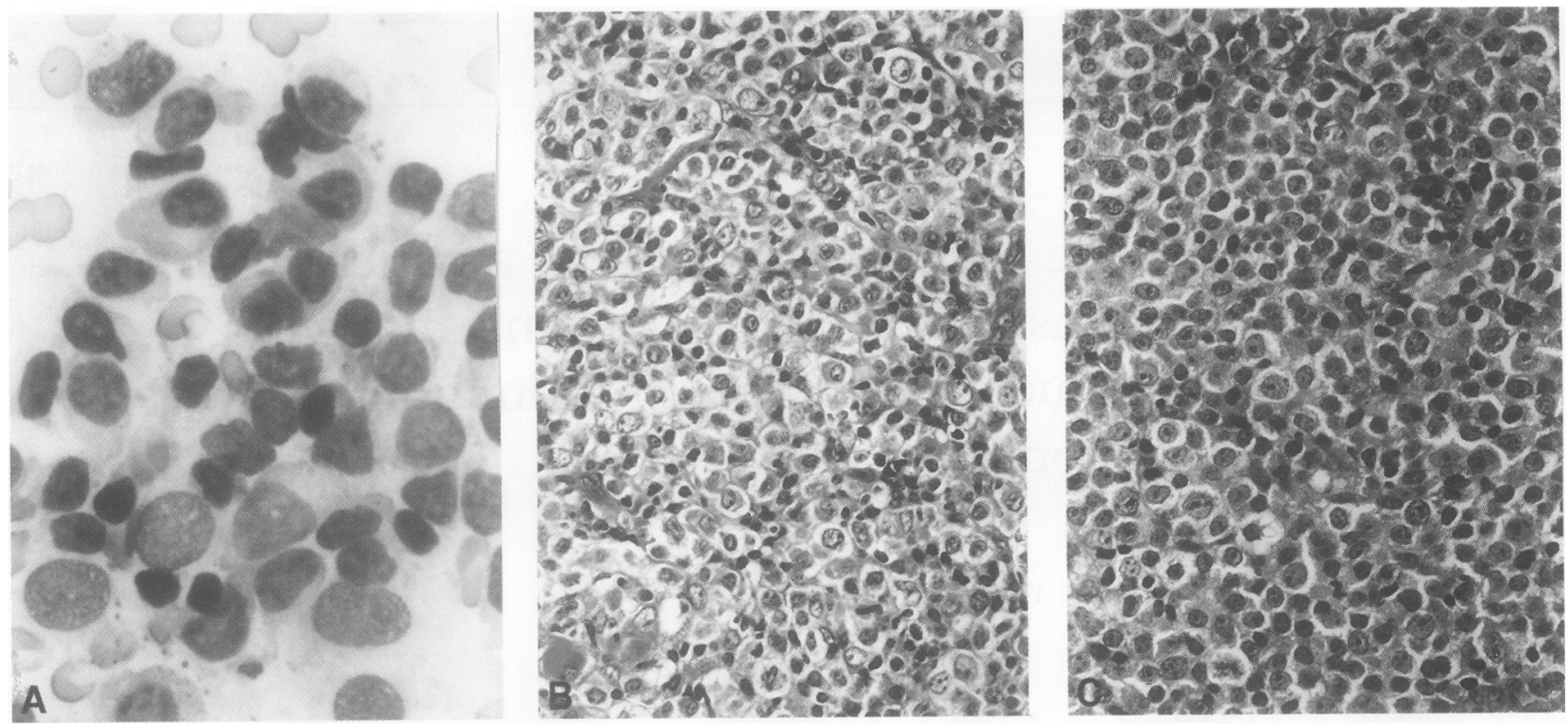

Figure 1 (A) Bone marrow histology at first admission demonstrating focal infiltration by lymphoplasmacytoid cells (May-Giemsa; original magnification $\times 400)$. (B) Lymph node biopsy at first admission showing obliteration of nodal architecture due to infiltration with vague nodularity of atypical cells (haematoxylin and eosin; original magnification $\times 100$ ). (C) Lymph node biopsy at second admission showing complete obliteration of nodal architecture due to diffuse infiltration of immunoblasts (haematoxylin and eosin; original magnification $\times 100$ ).

Blood biochemistry disclosed an elevated lactate dehydrogenase activity (840 IU/1). Serum immunoelectrophoresis showed a trace amount of monoclonal IgM ( $\kappa$ light chains) although its serum concentration was within normal range. Bone marrow studies disclosed a focal infiltration of lymphoplasmacytoid cells, although abnormal cells were not detected in peripheral blood. Immunoblastic lymphoma was detected on examination of a lymph node biopsy specimen taken from the neck. Waldenström's macroglobulinaemia was thought to have developed into immunoblastic lymphoma and the patient was treated with the CHOP (cyclophosphamide, hydroxydaunomycin, oncovin, and prednisolone) regimen following partial remission, but died one and a half years later.

Surface markers of abnormal cells in the peripheral blood were stained by an indirect immunofluorescence method using CD20 (B1), SmIg and PCA-1 monoclonal antibodies and analysed using flow cytometry. Paraffin wax sections of bone marrow and lymph node biopsy specimens were stained by the Streptavidin-biotin peroxidase (ABC) method using CD20 (L26), CD45RO (UCHL-1), anti-IgM, anti- $\kappa$, anti- $\lambda, \mathrm{MB}-1$, and MT-1 antibodies.

DNA was extracted from the paraffin wax blocks and amplified according to Wan $e t a l^{3}$ with minor modifications. Briefly, $1 \mu$ l extracted DNA was subjected to polymerase chain reaction (PCR) amplification with 2.5 units of Taq polymerase and with primers for $\mathrm{V}$ and $\mathrm{J}$ regions at a final concentration of $0.5 \mu \mathrm{M}$ in $100 \mu \mathrm{l}$ standard buffer. The denaturing step was carried out for 120 seconds at $94^{\circ} \mathrm{C}$, the annealing step for 120 seconds at $60^{\circ} \mathrm{C}$, and the extension step for 120 seconds at $72^{\circ} \mathrm{C}$ for 30 cycles using a thermal sequencer. The amplified DNA sample was diluted 1000-fold in water and added to a fresh PCR reaction mixture, followed by a second amplification sequence of 20 cycles. FR3A and LJH primers were used in the first 30 cycles, and FR3A and VLJH in the second 20.

For DNA sequencing, PCR products were subcloned into the PCRTM vector using TA cloning kits (Invitroge, San Diego, California, USA). Sequencing was performed using Taq dye primer cycle sequencing kits (Applied Biosystem, Tokyo, Japan).

\section{Results}

PERIPHERAL BLOOD AND BONE MARROW

In the smears of peripheral blood taken during the initial hospitalisation no one abnormal cell predominated; there were medium-sized lymphoid cells with basophilic cytoplasm and irregularly shaped nuclei with delicate chromatin and distinct nucleoli. Other cells resembled mature plasmacytes, although some had irregularly shaped nuclei. Surface marker analysis disclosed that the abnormal cells were strongly positive for PCA-1, weakly positive for CD20 and negative for SmIg. In bone marrow specimens accumulation of both mature and immature plasmacytes was observed at both the initial (fig 1A) and second hospitalisation.

\section{LYMPH NODES}

The first lymph node biopsy showed extensive, but incomplete, obliteration of nodal architecture due to proliferation of medium to large atypical lymphoid cells. The large cells had round nuclei while the medium-sized cells were lymphoplasmacytoid in nature (fig 1B). Most proliferating cells in the lymph node showed positive cytoplasmic staining with antisera to IgM $\kappa$ light chains and CD20. In 1993, the second lymph node biopsy specimen showed extensive and complete obliteration of the nodal architecture due to diffuse proliferation of immunoblasts (fig 2A). Some of 

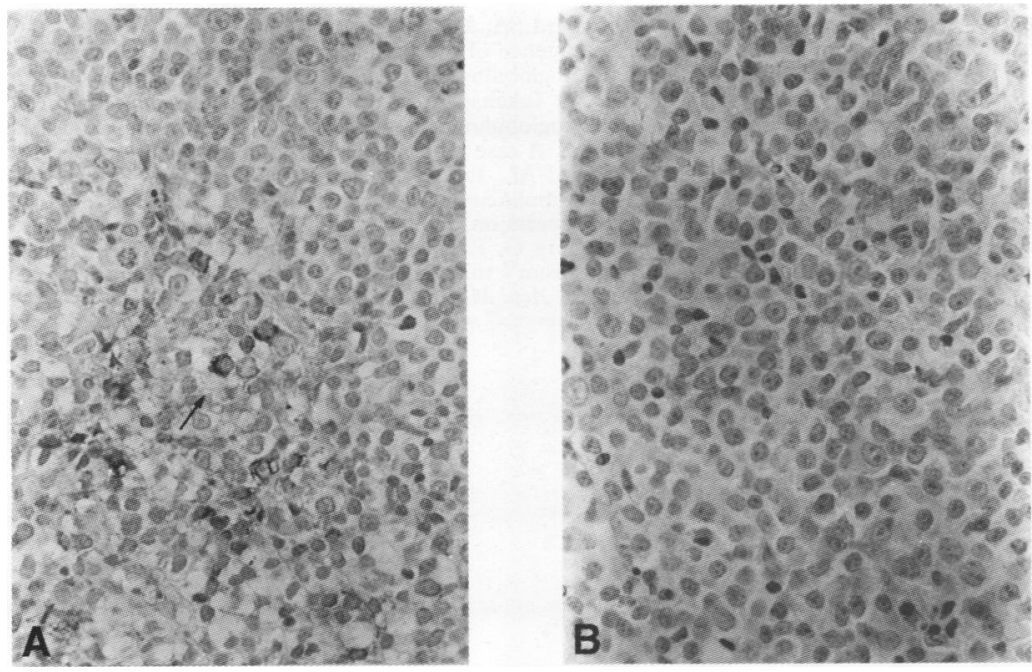

Figure 2 Same material as shown in fig $1 C$ stained using immunoperoxidase methods showing positive staining for $\kappa$ (arrow) $(A)$ and negative staining for $i(B)$ chains (original magnification $\times 100$ ). these conditions to be drawn. The genetic relation between these two malignancies has not been studied to date. In the case presented here histological and immunophenotypic evidence suggested malignant transformation of a single tumour clone, but on analysis of PCR products these tumours were found to have different $\mathrm{JH}$ rearrangements indicating that in this patient Waldenström's macroglobulinaemia did not progress to immunoblastic lymphoma.

Cytotoxic drugs have been implicated in the genesis of secondary malignancies in various conditions. As treatment with alkylating agents has become more common, more cases of Waldenström's macroglobulinaemia developing signs of high grade lymphoma have been reported. Österberg and Rausing ${ }^{10}$ reviewed 20 cases of Waldenström's macroglobulinaemia which progressed to "reticulum cell sarcoma" and found that 10 of these patients had received alkylating agents. The time lapse between the start of therapy and death ranged from one and a half to eight years. Our patient developed a high grade lymphoma four years after achieving complete remission following prolonged medication with melphalan and prednisolone. The time interval was consistent with a postchemotherapy lymphoma. Although in the paper by Österberg and Rausing only one patient had been treated with melphalan, that secondary malignancies often develop after treatment with this drug in cases of multiple myeloma suggests that it might be implicated in the generation of high grade malignant lymphomas. Other possibilities are that the immunodeficiency associated with Waldenström's macroglobulinaemia increases the risk of other malignancies by decreasing "immune surveillance", or that the initial abnormality that leads to the development of Waldenström's microglobulinaemia also underlies the development of immunoblastic lymphoma. As Epstein-Barr virus is often implicated in the genesis of lymphoid malignancies in immunodeficient patients, the significance of infection in the development of secondary malignancies in Waldenström's macroglobulinaemia merits further study.

In patients whose primary neoplasm developed drug resistance, a malignant progression of this clone is likely to result in a secondary malignancy resistant to the same drug(s). However, if the secondary malignancy is a different clone, then it may be drug sensitive and respond well. Therefore, clonal examination, including analysis of immunoglobulin gene rearrangements, would have an immediate practical application in the routine diagnostic laboratory.

1 Facon T, Brouillard M, Duhamel A, Morel P, Simon M, Jouet JP, et al. Prognostic factors in Waldenström's ma-
croglobulinemia: A report of 167 cases. $\mathcal{F}$ Clin Oncol 1993; 8roglobulin

2 Emmerich B, Pemsl M, Wust I, Berdel WE, Lechner S, Thiel $\mathrm{E}$, et al. Conversion of an IgM secreting immunocytoma to a high grade malignant lymphoma of immunoblastic type A case report. Blut 1983;46:81-4.

3 Wan JH, Trainor KJ, Brisco MJ, Morley AA. Monoclonality in $\mathrm{B}$ cell lymphoma detected in paraffin wax embedded sections using the polymerase chain reaction. $\mathcal{F}$ Clin Patho 1990;43:888-90. to immunoblastic lymphoma, ${ }^{78}$ which occurs in $1 \cdot 8^{1}$ to $10 \cdot 7 \%{ }^{9}$ of patients, but only a few cases have been described in the literature preventing conclusions about the pathogenesis of 
4 Richter MN. Generalized reticular cell sarcoma of lymph nodes associated with lymphatic leukemia. Am $f$ Pathol 1928:4:285-92.

5 Foon KA, Thirvengadam R, Saven A, Bernstein ZP, Gale $R P$. Genetic relatedness of lymphoid malignancies. Transformation of chronic lymphocytic leukemia as a model. Ann Intern Med 1993;119:63-73.

6 Ann Intern Med 1993;119:63-73. $M$, Bernard J. Malignant lymphoma supervening in chronic lymphocytic leukemia and related disorders. Richter's syndrome: a study of 25 cases. Cancer 1981;48: $1302-8$
7 Leonhard SA, Muhleman AF, Hurtubise PE, Martelo OJ Emergence of immunoblastic sarcoma in Waldenström's macroglobulinemia. Cancer 1980;45:3102-7.

8 Abe M, Takahashi K, Mori N, Kojima M. "Waldenström's macroglobulinemia" terminating in immunoblastic sarmacroglobulinemia" terminating in immuno

9 Lennard AL, Proctor J, Hamilton PJ. Waldenström's macroglobulinemia: a re-evaluation of the clinical syndrome forty years on. Hematol Rev 1987;1:243-58.

10 Österberg G, Rausing A. Reticulum cell sarcoma in Waldenström's macroglobulinemia after chlorambucil treatment. Acta Med Scand 1970;188:497-504. 